\title{
Robust Model Predictive Control for Discrete-time Fractional-order Systems ${ }^{\star}$
}

\author{
Pantelis Sopasakis ${ }^{\dagger, *}$, Sotirios Ntouskas* and Haralambos Sarimveis*
}

\begin{abstract}
In this paper we propose a tube-based robust model predictive control scheme for fractional-order discretetime systems of the Grünwald-Letnikov type with state and input constraints. We first approximate the infinitedimensional fractional-order system by a finite-dimensional linear system and we show that the actual dynamics can be approximated arbitrarily tight. We use the approximate dynamics to design a tube-based model predictive controller which endows to the controlled closed-loop system robust stability properties.
\end{abstract}

\section{Index Terms}

Fractional-order systems, Model Predictive Control, Grünwald-Letnikov derivative, Constraints satisfaction.

\section{INTRODUCTION}

\section{A. Background and Motivation}

Non-integer order derivatives and integrals (known as fractional) are natural extensions of the standard integerorder ones which enjoy certain favourable essential properties; for example they are linear operators, preserve analyticity, and have the semigroup property [1], [2]. However, fractional derivatives are non-local operators, that is, unlike their integer-order counterparts, they cannot be evaluated at a points $x$ solely by knowing how the function behaves in a neighbourhood of this point [1].

Their popularity in pharmacokinetics and pharmacodynamics is particularly interesting. The work of Kytariolos et al. introduced fractional dynamics in the field of pharmacokinetic modelling pointing out the main reasons for the failure of the classical in-vitro-in-vivo correlations theory [3]. Often, non-linearities, anomalous diffusion, deep tissue trapping, diffusion across fractal manifolds, synergistic and competitive action and a great many other factors give rise to fractional-order pharmacokinetics [4]. Such fractional pharmacokinetic dynamics can be cast as physiologically-based pharmacokinetic models (PBPK) (see [5]) where the mass balance equations are properly rewritten using fractional-order derivatives. Recently, it seems that there is increasing attention on modelling and control of such systems [5]-[7], especially in presence of state and input constraints.

Overall, fractional-order dynamical systems have been proven to be powerful modelling tools used to describe dynamics with infinite memory and are becoming increasingly popular as it is becoming evident that they can compactly describe rather complex dynamics.

Very recently, fractional systems were combined with the model predictive control (MPC) framework [8]-[12]; this seems to be a very active and emerging topic of research and has a great potential for applications [13], [14]. Domek proposed an MPC setting for Takagi-Sugeno fuzzy fractional-order systems [15] and switched MPC of fractional-order systems [16]. Deng et al. [10] proposed a predictive control scheme for the power regulation of a solid oxide fuel cell. A common denominator of all approaches in the literature is that they approximate the actual fractional dynamics by integer-order dynamics and design controllers for the approximate system using standard techniques. Although, these approaches seem to work in practice, no theoretical guarantee is provided for the stability of the closed-loop system. Additionally, MPC is often employed in order to address constraints on the state and input of the system, but no theoretical guarantees for their satisfaction exist in the literature. The main contribution of this paper is a design methodology for a model predictive control scheme which steers

\footnotetext{
$\dagger$ IMT Institute for Advanced Studies Lucca, Piazza San Ponziano 6, 55100 Lucca, Italy. Email: pantelis.sopasakiseimtlucca.it.

* National Technical University of Athens (NTUA), School of Chemical Engineering, 9 Heroon Polytechneiou Street, 15780 Zografou Campus, Athens, Greece. Emails: \{sotdouska, hsarimv\}ecentral.ntua.gr.

* This work was funded by project $11 \Sigma Y$ N.10.1152, which is co-financed by the European Union and Greece, Operational Program "Competitiveness \& Entrepreneurship", NSFR 2007-2013 in the context of GSRT-National action "Cooperation".
} 
the system's state towards a small neighbourhood of the origin while it guarantees the satisfaction of state and input constraints.

Two approaches can be identified in the literature in regard to the stability analysis of discrete-time fractional systems. The first one considers the stability of a finite-dimensional linear time-invariant system, known as practical stability, but fails to provide conditions for the actual fractional-order system to be (asymptotically) stable [17], [18]. This approach is tacitly pursued in many applied papers [12], [19]. On the other hand, fractional systems can be treated as infinite-dimensional systems for which various stability conditions can be derived (See for example [20, Thm. 2]), but conditions are difficult to verify in practice let alone to use for the design of model predictive controllers.

In this paper we discretise linear continuous-time fractional dynamics using the Grünwald-Letnikov scheme which leads to infinite-dimensional linear systems. We use a finite-dimensional approximation to arrive at a linear time-invariant system with an additive uncertainty term which casts the discrepancy with the infinite-dimensional system. We then introduce a tube-based MPC control scheme which is known to steer the state to a neighbourhood of the origin which can become arbitrarily small as the complexity of the approximation of the fractional-order system increases. In our analysis, we consider both state and input constraints which we show that are respected by the MPC-controlled system.

\section{B. Mathematical preliminaries}

For the paper to be self-contained, in this section we introduce some notation and definitions that will be useful throughout the whole paper. Let $\mathbb{N}, \mathbb{R}^{n}, \mathbb{R}_{+}, \mathbb{R}^{m \times n}$ denote the set of non-negative integers, the set of column real vectors of length $n$, the set of non-negative numbers and the set of $m$-by- $n$ real matrices respectively. For any nonnegative integers $k_{1} \leq k_{2}$ the finite set $\left\{k_{1}, \ldots, k_{2}\right\}$ is denoted by $\mathbb{N}_{\left[k_{1}, k_{2}\right]}$. Let $x$ be a sequence of real vectors of $\mathbb{R}^{n}$. The $k$-th vector of the sequence is denoted by $x_{k}$ and its $i$-th element is denoted by $x_{k, i}$. We denote by $\mathcal{B}_{\epsilon}^{n}=\left\{x \in \mathbb{R}^{n}:\|x\|<\epsilon\right\}$ the open ball of $\mathbb{R}^{n}$ with radius $\epsilon$ and we use the shorthand $\mathcal{B}^{n}=\mathcal{B}_{1}^{n}$. We define the point-to-set distance of a point $z \in X$ from $A$ as $\operatorname{dist}(z, A)=\inf _{a \in A}\|z-a\|$.

The space of bounded real sequences is denoted by $\ell^{\infty}$ and is a Banach space with norm $\|z\|_{\infty}=\sup _{k \in \mathbb{N}}\left|z_{k}\right|$. We define the space $\ell_{n}^{\infty}$ of all sequences of real $n$-vectors $z$ so that $\left(z_{k, i}\right)_{k} \in \ell^{\infty}$ for $i \in \mathbb{N}_{[1, n]}$ which, equipped with the norm $\|z\|_{\infty}=\max _{i=1, \ldots, n}\left\|z_{k, i}\right\|_{\infty}$, becomes a Banach space.

Let $\Gamma$ be a topological real vector space and $A, B \subseteq \Gamma$. For $\lambda \in \mathbb{R}$ we define the scalar product $\lambda C=\{\lambda c$ : $c \in C\}$ and the Miknowski sum $A \oplus B=\{a+b: a \in A, b \in B\}$. The Minkowski sum of a finite family of sets $\left\{A_{i}\right\}_{i=1}^{K}$ will be denoted by $\bigoplus_{i=1}^{K} A_{i}$. The Minkowski sum of a sequence of sets $\left\{A_{i}\right\}_{i \in \mathbb{N}}$ is denoted as $\bigoplus_{i \in \mathbb{N}} A_{i}$ and is defined as the Painlevé-Kuratowski limit (see [21]) of $\bigoplus_{i=1}^{K} A_{i}$ as $K \rightarrow \infty$. The Pontryagin difference between two sets $A, B \subseteq \Gamma$ is defined as $A \ominus B=\{a \in A: a+b \in A, \forall b \in B\}$. A set $C$ is called balanced if for every $x \in C,-x \in C$.

\section{FRACTIONAL-ORDER SYSTEMS}

\section{A. Discrete-time fractional operators}

Let $x: \mathbb{R} \rightarrow \mathbb{R}^{n}$ be a uniformly bounded function, i.e., there is a $M>0$ so that $\|x(t)\| \leq M$ for all $t \in \mathbb{R}$. The backward Grünwald-Letnikov fractional-order difference of $x$ of order $\alpha>0$ and step size $h>0$ is defined as the linear operator [13] ${ }_{B} \Delta_{h}^{\alpha}: \ell_{n}^{\infty} \rightarrow \ell_{n}^{\infty}$ :

$$
{ }_{B} \Delta_{h}^{\alpha} x(t)=\sum_{j=0}^{\infty}(-1)^{j}\left(\begin{array}{l}
\alpha \\
j
\end{array}\right) x(t-j h),
$$

and its forward counterpart is defined as ${ }_{F} \Delta_{h}^{\alpha} x(t)={ }_{B} \Delta_{h}^{\alpha} x(t+h)$, or

$$
{ }_{F} \Delta_{h}^{\alpha} x(t)=\sum_{j=0}^{\infty}(-1)^{j}\left(\begin{array}{c}
\alpha \\
j
\end{array}\right) x(t+(1-j) h),
$$

where $\left(\begin{array}{l}\alpha \\ 0\end{array}\right)=1$ and for $j \in \mathbb{N}, j>0$

$$
\left(\begin{array}{l}
\alpha \\
j
\end{array}\right)=\prod_{i=0}^{j-1} \frac{\alpha-i}{i+1}
$$


Define

$$
c_{j}^{\alpha}=(-1)^{j}\left(\begin{array}{c}
\alpha \\
j
\end{array}\right)
$$

and notice for all $j \in \mathbb{N}$ that $\left|c_{j}^{\alpha}\right| \leq \alpha^{j} / j$ !, thus, the sequence $\left(c_{j}^{\alpha}\right)_{j}$ is absolutely summable and, because of the uniform boundedness of $x$, the series in (2) converges, therefore, $\Delta_{h}^{\alpha}$ is well-defined. It is worth noticing that at time $t$ and for non-integer orders $\alpha$ the whole history of $x$ is needed in order to estimate $\Delta_{h}^{\alpha} x(t)$.

The Grünwald-Letnikov difference operator gives rise to the Grünwald-Letnikov derivative of order $\alpha$ which is defined as

$$
D^{\alpha} x(t)=\lim _{h \rightarrow 0} \frac{{ }_{F} \Delta_{h}^{\alpha} x(t)}{h^{\alpha}}=\lim _{h \rightarrow 0} \frac{{ }_{B} \Delta_{h}^{\alpha} x(t)}{h^{\alpha}},
$$

provided that both limits exist. This derivative is then used to describe fractional-order dynamical systems with state $x: \mathbb{R} \rightarrow \mathbb{R}^{n}$ and input $u: \mathbb{R} \rightarrow \mathbb{R}^{m}$ as follows:

$$
\sum_{i=0}^{l} A_{i} D^{\alpha_{i}} x(t)=\sum_{i=0}^{r} B_{i} D^{\beta_{i}} u(t),
$$

where $l, r \in \mathbb{N}, A_{i}$ are $B_{i}$ are matrices of opportune dimensions, all $\alpha_{i}$ and $\beta_{i}$ are nonnegative, and by convention $D^{0} x(t)=x(t)$ for any $x$.

In an Euler discretisation fashion we approximate the $D^{\alpha}$ in (6) using either $h^{-\alpha}{ }_{F} \Delta_{h}^{\alpha}$ or $h^{-\alpha}{ }_{B} \Delta_{h}^{\alpha}$ for a fixed step size $h$. In particular, we use the forward operator for the derivatives of the state and the backward one for the input variables. For convenience, let us define $x_{k}=x(k h)$ and $u_{k}=u(k h)$ for $k \in \mathbb{Z}$; the discretisation of (6) becomes

$$
\sum_{i=0}^{l} \bar{A}_{i B} \Delta_{h}^{\alpha_{i}} x_{k+1}=\sum_{i=0}^{r} \bar{B}_{i B} \Delta_{h}^{\beta_{i}} u_{k},
$$

with $\bar{A}_{i}=h^{-\alpha_{i}} A_{i}$ and $\bar{B}_{i}=h^{-\beta_{i}} B_{i}$. The involvement of infinite-dimensional operators in the system dynamics deem these systems computationally intractable and call for approximation methods for their simulation and the design of feedback controllers.

In what follows, we will approximate (7) by a finite-dimensional state-space system treating the approximation as a bounded additive disturbance. We then propose a control setting which guarantees robust stability properties for (7).

\section{B. Finite-dimension approximation}

It turns out that discrete-time fractional-order dynamical systems are systems with infinite memory and encompass an infinite number of state variables. Consequently, the results of standard control theory cannot be applied directly. For this purpose, we introduce the following truncated Grünwald-Letnikov difference operator or length $\nu$

$$
\Delta_{h, \nu}^{\alpha} x_{k}=\sum_{j=0}^{\nu} c_{j}^{\alpha} x_{k-j},
$$

and the approximate finite-dimensional variant of (7), for $\nu \geq 1$, becomes

$$
\sum_{i=0}^{l} \bar{A}_{i} \Delta_{h, \nu}^{\alpha_{i}} x_{k+1}=\sum_{i=0}^{r} \bar{B}_{i} \Delta_{h, \nu-1}^{\beta_{i}} u_{k} .
$$




\section{State space representation}

System (9) can be written in state space format as a linear time-invariant (LTI) system with a proper choice of state variables $\tilde{x}_{k}$ as we shall explain in this section. In the common case where the right-hand side of (9) is of the simple form $B u_{k}$, it is straightforward to recast the system in state-space form. Here, we study the more general case of equation (9), which can be written in the form

$$
\sum_{j=0}^{\nu} \hat{A}_{j} x_{k-j+1}=\sum_{j=0}^{\nu} \hat{B}_{j} u_{k-j}
$$

with

$$
\begin{aligned}
& \hat{A}_{j}=\sum_{i=0}^{l} \bar{A}_{i} c_{j}^{\alpha_{i}}, \\
& \hat{B}_{j}=\sum_{i=0}^{r} \bar{B}_{i} c_{j}^{\beta_{i}},
\end{aligned}
$$

for $j \in \mathbb{N}_{[0, \nu]}$. We hereafter assume that matrix $\hat{A}_{0}$ is nonsingular. Then, defining

$$
\begin{aligned}
& \tilde{A}_{j}=-\hat{A}_{0}^{-1} \hat{A}_{j}, \\
& \tilde{B}_{j}=\hat{A}_{0}^{-1} \hat{B}_{j},
\end{aligned}
$$

the dynamic equation (10) becomes

$$
x_{k+1}=\sum_{j=0}^{\nu-1} \tilde{A}_{j} x_{k-j}+\sum_{j=1}^{\nu} \tilde{B}_{j} u_{k-j}+\tilde{B}_{0} u_{k} .
$$

This can be written in state space form with state variable $\tilde{x}_{k}=\left(x_{k}, x_{k-1}, \ldots, x_{k-\nu+1}, u_{k-1}, \ldots, u_{k-\nu}\right)^{\prime}$, as

$$
\tilde{x}_{k+1}=A \tilde{x}_{k}+B u_{k} .
$$

System (14) is an ordinary finite-dimensional LTI system which will be used in the next section to formulate a model predictive control problem. Throughout the rest of the paper we assume that the pair $(A, B)$ is stabilisable.

\section{Model Predictive Control}

\section{A. Control-oriented modelling}

Inevitably, the use of the truncated difference operator $\Delta_{h, \nu}^{\alpha}$ introduces some error in the system dynamics. In particular, the fractional-order difference operator ${ }_{B} \Delta_{h}^{\alpha}$ can be written as

$$
{ }_{B} \Delta_{h}^{\alpha}=\Delta_{h, \nu}^{\alpha}+R_{h, \nu}^{\alpha},
$$

where $R_{h, \nu}^{\alpha}: \ell_{n}^{\infty} \rightarrow \ell_{n}^{\infty}$ is the operator $R_{\nu}^{\alpha}\left(x_{k}\right)=h^{-\alpha} \sum_{j=\nu+1}^{\infty} c_{j}^{\alpha} x_{k-j}$. Let $X$ be a compact convex subset or $\mathbb{R}^{n}$ containing 0 in its interior and at time $k$ assume that $x_{k-j} \in X$ for all $j \in \mathbb{N}$. Then, given that $x_{k-j} \in X$ for all $j \in \mathbb{N}$,

$$
R_{h, \nu}^{\alpha}\left(x_{k}\right) \in \bigoplus_{j=\nu+1}^{\infty} c_{j}^{\alpha} X .
$$

The fractional dynamics (7) can now be written in terms of $\tilde{x}$ (cf. (14)) as the linear uncertain system

$$
\tilde{x}_{k+1}=A \tilde{x}_{k}+B u_{k}+G d_{k},
$$

where $d_{k}$ is a additive disturbance term (which depends on $x_{k-\nu-j}$ and $u_{k-\nu-j}$ for $j \in \mathbb{N}$ ) with $G=\left[\begin{array}{lllll}I & 0 & \ldots & 0\end{array}\right]^{\prime}$. Assume that $u_{k-j} \in U$ for $j=1,2, \ldots$ and $x_{k-j} \in X$ for $j \in \mathbb{N}$, where $X$ and $U$ are convex compact sets containing 0 in their interiors. Then, $d_{k}$ is bounded in a compact set $D_{\nu}$ given by

$$
D_{\nu}=D_{\nu}^{x} \oplus D_{\nu}^{u}
$$


where

$$
\begin{aligned}
D_{\nu}^{x} & =\bigoplus_{i=0}^{l}-\hat{A}_{0}^{-1} \bar{A}_{i} \bigoplus_{j=\nu+1}^{\infty} c_{j}^{\alpha_{i}} X, \\
D_{\nu}^{u} & =\bigoplus_{i=0}^{r} \hat{A}_{0}^{-1} \bar{B}_{i} \bigoplus_{j=\nu+1}^{\infty} c_{j}^{\beta_{i}} U .
\end{aligned}
$$

Under the prescribed assumptions, both $D_{\nu}^{x}$ and $D_{\nu}^{u}$ are compact sets, therefore $D_{\nu}$ will also be compact.

Recall that for a balanced set $C \subseteq \mathbb{R}^{n}$ and scalars $\lambda_{1}, \lambda_{2}$ it is $\lambda_{1} C \oplus \lambda_{2} C=\left(\left|\lambda_{1}\right|+\left|\lambda_{2}\right|\right) C$. In case $X$ and $U$ are balanced sets, the above expressions for $D_{\nu}^{x}$ and $D_{\nu}^{u}$ can be simplified. First, for $\nu \in \mathbb{N}$, we define the function $\Psi_{\nu}: \mathbb{R}_{+} \rightarrow \mathbb{R}_{+}$as the following convergent series:

$$
\Psi_{\nu}(\alpha)=\sum_{j=\nu+1}^{\infty}\left|c_{j}^{\alpha}\right| .
$$

Then, $D_{\nu}^{x}$ is written as the finite Minkowski sum

$$
D_{\nu}^{x}=\bigoplus_{i \in \mathbb{N}_{[0, l]}}-\hat{A}_{0}^{-1} \bar{A}_{i} \Psi_{\nu}\left(\alpha_{i}\right) X
$$

and of course the same simplification applies to $D_{\nu}^{u}$ if $U$ is a balanced set. Notice that the computation of $D_{\nu}^{x}$ by (21) boils down to determining a finite Minkowski sum, which is possible when constraints are polytopic [22], while overapproximations exists when they are ellipsoidal [23].

The size of $D_{\nu}$ is controlled by the choice of $\nu ; D_{\nu}$ can become arbitrarily small provided that a sufficiently large $\nu$ is chosen. Notice also that $D_{\nu} \rightarrow\{0\}$ as $\nu \rightarrow \infty$. In light of (17), the fractional system can be controlled by standard methods of robust control such as min-max [24] or tube-based MPC [25] as we do in this paper. In what follows, we elaborate on how the tube-based MPC methodology can be applied for the control of fractional-order systems.

\section{B. Tube-based Model Predictive Control}

MPC is an optimisation-based control methodology where at each time instant a performance index is optimised along an horizon of future time instants using a discrete-time model of the controlled process taking into account the constraints on the state and input variables.

MPC, by solving an optimisation problem, produces a sequence of control actions whose first element in applied to the system as input while all other elements are discarded and the same procedure is repeated at every time instant; this control scheme defines the receding horizon control approach [25].

Here, we require that the state and input variables are constrained in the sets $X \subseteq \mathbb{R}^{n}$ and $U \subseteq \mathbb{R}^{m}$ respectively, both convex, compact and contain the origin in their interior. The constraints are written as follows:

$$
\begin{aligned}
& \tilde{x}_{k} \in \tilde{X}, \\
& u_{k} \in U,
\end{aligned}
$$

for all $k \in \mathbb{N}$ and where $\tilde{X}=X^{\nu} \times U^{\nu}$, i.e., $\tilde{x}=\left(x_{k}, x_{k-1}, \ldots, x_{k-\nu+1}, u_{k-1}, \ldots, u_{k-\nu}\right)^{\prime} \in \tilde{X}$ if and only if $x_{k-i} \in X$ for $i \in \mathbb{N}_{[0, \nu-1]}$ and $u_{k-i} \in U$ for all $i \in \mathbb{N}_{[1, \nu]}$. Typically, in MPC $\tilde{X}$ and $U$ can be polytopes or ellipsoids, but for our analysis no particular assumptions on $X$ and $U$ need to be imposed.

The tube-based MPC scheme is illustrated in Figure 1. The fractional-order system is controlled by an input $u$ which is computed according to

$$
u_{k}=v_{k}+K e_{k},
$$

where $v_{k}$ is a control action computed by the tube-based MPC controller and $e_{k}$ is defined as the deviation between the actual system state and the response of the nominal system. In particular the nominal dynamics in terms of the nominal state $\tilde{z}_{k}$ with input $v_{k}$ is

$$
\tilde{z}_{k}=A \tilde{z}_{k-1}+B v_{k-1},
$$




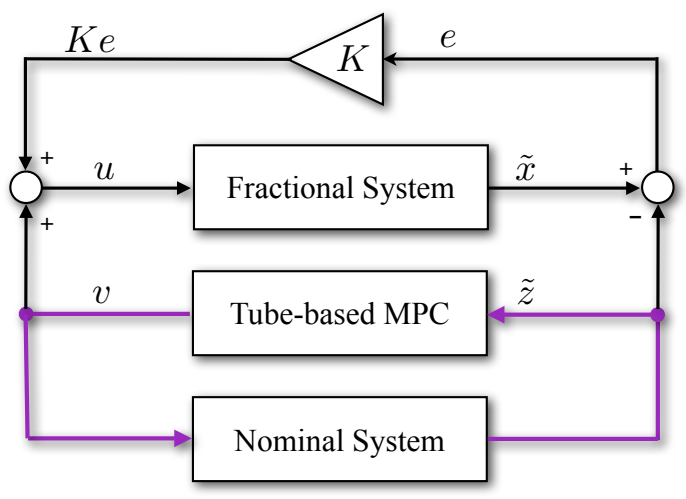

Fig. 1: Tube-based MPC for fractional order systems. The nominal system is a finite dimension LTI system in discrete-time.

and $e_{k}=\tilde{x}_{k}-\tilde{z}_{k}$. The deviation variable $e$ follows the stable dynamics $e_{k+1}=A_{K} e_{k}+G d_{k}$. Matrix $K$ in (23) is chosen so that $A_{K}=A+B K$ is strongly stable. We define

$$
S_{k}=\bigoplus_{i=0}^{k} A_{K}^{i} G D_{\nu}
$$

The set $S_{\infty}$, defined as the limit $S_{\infty}=\lim _{k \rightarrow \infty} S_{k}$, is well-defined (the limit exists), is compact, and is positive invariant for the deviation dynamics $e_{k+1}=A_{K} e_{k}+G d_{k}$. In what follows, $S_{\infty}$ will be assumed to contain the origin in its interior. For the needs of tube-based MPC, any over-approximation of $S_{\infty}$ may be used instead - a comprehensive discussion on various computational aspects regarding $S_{\infty}$ can be found in [25].

Having chosen $\tilde{z}_{0}=\tilde{x}_{0}$, it is $\tilde{x}_{k} \in\left\{\tilde{z}_{k}\right\} \oplus S_{\infty}$ for all $k \in \mathbb{N}$. This implies that constraint (22a) is satisfied if $\tilde{z}_{k} \in X \ominus S_{\infty}$ and constraint (22b) is satisfied if $v_{k} \in U \ominus K S_{\infty}$. These constraints will then be involved in the formulation of the MPC problem which produces the control actions $v_{k}=v_{k}\left(\tilde{z}_{k}\right)$.

The MPC problem amounts to the minimisation of a performance index $V_{N}$ along an horizon of future time instants, known as the prediction horizon, given the initial state at time $k, \tilde{z}_{k}$. Let $N$ be the prediction horizon. We use the notation $\tilde{z}_{k+i \mid k}$ for the predicted state of the nominal system at time $k+i$ using feedback information at time $k$. Let $\mathbf{v}_{k}=\left\{v_{k+i \mid k}\right\}_{i \in \mathbb{N}_{[0, N-1]}}$ be a sequence of input values and $\left\{\tilde{z}_{k+i \mid k}\right\}_{i \in \mathbb{N}_{[1, N]}}$ the corresponding predicted states obtained by (24), i.e., it is $\tilde{z}_{k+i+1 \mid k}=A \tilde{z}_{k+i \mid k}+B v_{k+i \mid k}$ for $i \in \mathbb{N}_{[0, N-1]}$. We introduce following performance index $V_{N}: \mathbb{R}^{\bar{n}} \times \mathbb{R}^{m N} \rightarrow \mathbb{R}_{+}$given the current state of the system $\tilde{z}_{k \mid k}=\tilde{z}_{k}$ :

$$
V_{N}\left(\tilde{z}_{k \mid k}, \mathbf{v}_{k}\right)=V_{f}\left(\tilde{z}_{k+N \mid k}\right)+\sum_{i=0}^{N-1} \ell\left(\tilde{z}_{k+i \mid k}, v_{k+i \mid k}\right),
$$

where $\ell$ and $V_{f}$ are typically quadratic functions. We assume that

$$
\ell(z, v)=z^{\prime} Q z+v^{\prime} R v
$$

where $Q$ is symmetric, positive semidefinite and $R$ is symmetric positive definite and

$$
V_{f}(z)=z^{\prime} P z,
$$

where $P$ is symmetric and positive definite. The following constrained optimisation problem is then solved:

$$
\mathbb{P}_{N}: V_{N}^{\star}\left(\tilde{z}_{k}\right)=\min _{\mathbf{v}_{k} \in \mathcal{V}_{N}\left(\tilde{z}_{k}\right)} V_{N}\left(\tilde{z}_{k}, \mathbf{v}_{k}\right)
$$


with

$$
\mathcal{V}_{N}\left(\tilde{z}_{k}\right)=\left\{\mathbf{v} \mid \begin{array}{l}
\tilde{z}_{k+i+1 \mid k}=A \tilde{z}_{k+i \mid k}+B v_{k+i \mid k}, \\
\forall i \in \mathbb{N}_{[0, N-1]} \\
\tilde{z}_{k \mid k}=\tilde{z}_{k} \\
\tilde{z}_{k+i \mid k} \in \tilde{X} \ominus S, \forall i \in \mathbb{N}_{[1, N]} \\
v_{k+i \mid k} \in U \ominus K S, \forall i \in \mathbb{N}_{[0, N-1]} \\
\tilde{z}_{k+N \mid k} \in \tilde{X}_{f}
\end{array}\right\},
$$

where $S$ is any over-approximation of $S_{\infty}$, i.e., $S \supseteq S_{\infty}$ and $\tilde{X}_{f} \subseteq \tilde{X}$ is the terminal constraints set. In what follows we always assume that $\tilde{X} \ominus S$ and $U \ominus K S$ are nonempty sets with the origin in their interior. In regard to the terminal cost function $V_{f}$ and the terminal constraints set $\tilde{X}_{f}$ we assume the following:

Assumption 1: $V_{f}$ and $\tilde{X}_{f}$ satisfy the standard stabilising conditions A1-A4 in [26].

Remark 2: Typically, matrix $P$ in (28) is chosen to be the (unique) solution of the discrete-time algebraic Riccatti equation $P=(A+B F)^{\prime} P(A+B F)+Q+F^{\prime} R F$ with $F=-\left(B^{\prime} P B+R\right)^{-1} B^{\prime} P A$ and $\tilde{X}_{f}$ to the maximal invariant constraint admissible set for the system $\tilde{z}_{k+1}=(A+B F) \tilde{z}_{k}$. Alternatively, one may choose $\tilde{X}_{f}$ to be an ellipsoid of the form $\tilde{X}_{f}=\left\{z: V_{f}(z) \leq \gamma\right\}$ and $\gamma>0$ is chosen so that $\tilde{X}_{f} \subseteq \tilde{X}$ and $K \tilde{X}_{f} \subseteq U$. The solution of $\mathbb{P}_{N}$, namely the optimiser

$$
v^{\star}\left(\tilde{z}_{k}\right)=\underset{\mathbf{v}_{k} \in \mathcal{V}_{N}\left(\tilde{z}_{k}\right)}{\operatorname{argmin}} V_{N}\left(\tilde{z}_{k}, \mathbf{v}_{k}\right),
$$

is a sequence $\left\{v_{0}^{\star}\left(\tilde{z}_{k}\right), v_{1}^{\star}\left(\tilde{z}_{k}\right), \ldots, v_{N-1}^{\star}\left(\tilde{z}_{k}\right)\right\}$ and its first element $v_{0}^{\star}\left(\tilde{z}_{k}\right)$, in a receding horizon control fashion, defines the control law

$$
\kappa_{N}\left(\tilde{z}_{k}\right)=v_{0}^{\star}\left(\tilde{z}_{k}\right)
$$

and the control action applied to the system is computed as $\rho\left(\tilde{z}_{k}, \tilde{x}_{k}\right)=\kappa_{N}\left(\tilde{z}_{k}\right)+K\left(\tilde{x}_{k}-\tilde{z}_{k}\right)$ and the closed-loop system is now expressed in terms of both $\tilde{z}_{k}$ and $\tilde{x}_{k}$ as

$$
\begin{aligned}
\tilde{x}_{k+1} & =A \tilde{x}_{k}+B \rho\left(\tilde{z}_{k}, \tilde{x}_{k}\right)+G d_{k}, \\
\tilde{z}_{k+1} & =A \tilde{z}_{k}+B \kappa_{N}\left(\tilde{z}_{k}\right) .
\end{aligned}
$$

The stability properties of the controlled system are studied for the composite system (33) with state variable $(\tilde{x}, \tilde{z})$.

\section{Stability results}

In this section we discuss the stability properties of the controlled closed-loop system presented previously. In robust control the following stability definition is usually employed [25, Def. B4]:

Definition 3 (Asymptotic Stability): Let $S$ be a closed nonempty set, positive invariant for $x_{k+1}=f\left(x_{k}\right)$. The set $S$ is locally stable for the aforementioned system if for all $\epsilon>0$ there is $\delta>0$ so that $\operatorname{dist}\left(x_{k}, S\right)<\epsilon$ for all $k \in \mathbb{N}$ whenever $\operatorname{dist}\left(x_{0}, S\right)<\delta$. If in addition, $\lim _{k} \operatorname{dist}\left(x_{k}, S\right)=0$, we say that $S$ is locally asymptotically stable.

A stronger form of stability, namely exponential stability is defined as follows:

Definition 4 (Exponential stability): The set $S$ is locally exponentially stable for $x_{k+1}=f\left(x_{k}\right)$ if there are $\eta>0, c>0$ and $\gamma \in(0,1)$ so that $\operatorname{dist}\left(x_{k}, A\right) \leq c \operatorname{dist}\left(x_{0}, A\right) \gamma^{k}$ for all $k \in \mathbb{N}$ whenever $\operatorname{dist}\left(x_{0}, A\right)<\eta$. The following result, which readily follows from [25, Prop. 3.15], states that the system's state converges towards $S_{\infty}$ exponentially provided that $S=S_{\infty}$ is used in the formulation of the MPC problem.

Proposition 5 (Exponential stability): Assume that the MPC control law $\kappa_{N}$ stabilises the nominal dynamical system (33b). The set $S_{\infty} \times\{0\}$ is locally exponentially stable for system (33) with region of attraction $\left(Z_{N} \oplus\right.$ $\left.S_{\infty}\right) \times Z_{N}$, where $Z_{N}$ is the domain of $\mathcal{V}_{N}$, i.e., $Z_{N}=\left\{x: \mathcal{V}_{N}(x) \neq \varnothing\right\}$.

In addition, the controlled trajectory of the system's state $x_{k}$ and input $u_{k}$ satisfy constraints (22) at all time instants $k \in \mathbb{N}$.

Notice that $S_{\infty}$ can become arbitrarily small with an appropriate choice of $\nu$ and the system's state can be steered this way very close to the origin, although, in practice large values of $\nu$ should be avoided to limit the complexity of $\mathbb{P}_{N}$. 


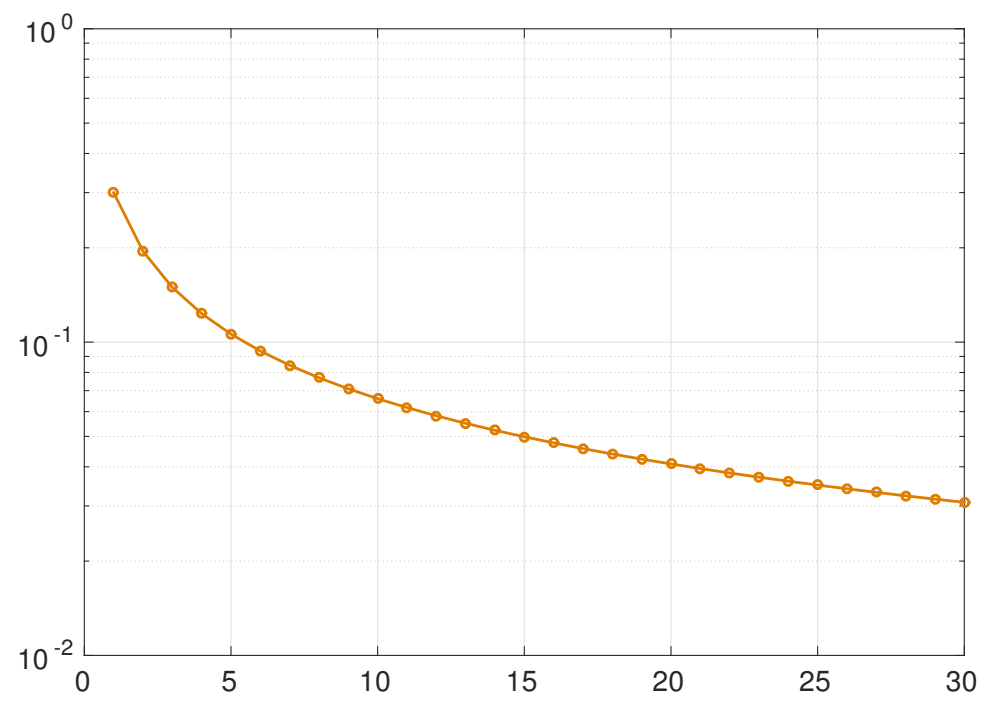

Fig. 2: Dependence of $\Psi_{\nu}(\alpha)$ on $\nu$ for $\alpha=0.7$ and selection of a reasonably and adequately large approximation order $\nu$.

\section{NUMERICAL EXAMPLE}

We apply the proposed methodology to the following fractional-order system

$$
D^{\alpha} x=\left[\begin{array}{cc}
0 & 1 \\
-1 & 0.3
\end{array}\right] x+\left[\begin{array}{c}
0 \\
-0.5
\end{array}\right] u,
$$

with $x \in \mathbb{R}^{2}$ and $u \in \mathbb{R}$ and $\alpha=0.7$. We discretise the system with sampling period $h=0.05$ and we use $\nu=20$ based on Figure 2 so that $\Psi_{\nu}(\alpha)$ is adequately small. In particular, we have $\Psi_{20}(0.7)=0.0408$. This way, we derive a discrete-time LTI system of the form $\tilde{x}_{k}=A \tilde{x}_{k-1}+B u_{k-1}$ as in Section II-B. The system state and input are subject to the constraints

$$
\begin{gathered}
-\left[\begin{array}{l}
0.5 \\
0.5
\end{array}\right] \leq x_{k} \leq\left[\begin{array}{l}
0.5 \\
0.5
\end{array}\right], \\
-0.15 \leq u_{k} \leq 0.15
\end{gathered}
$$

The terminal cost $V_{f}$ and the terminal constraints set $\tilde{X}_{f}$ were computed according so that the stabilising conditions A1-A4 of [26] are satisfied. In particular $\tilde{X}_{f}$ was chosen to be a sublevel set of $V_{f}$ as explained in Remark 2. The prediction horizon was chosen to be $N=20$ and the closed-loop state and input trajectory of the controlled system are presented in Figure 3.

The controller was implemented in MATLAB using Yalmip [27] and the solver mosek (https://www.mosek.com/). Out of 100 randomly selected (feasible) inital points, the MPC optimisation problem was solved on average in 15.5ms (maximum $22.3 \mathrm{~ms}$ ).

\section{CONCLusions AND Future Work}

In this paper we proposed a robust MPC scheme for fractional systems with guaranteed satisfaction of state and input constraints where the state converges exponentially fast to a neighbourhood of the origin whose size is controlled by the order of approximation $\nu$. The underlying optimisation problem we have to solve is a quadratic problem which can be solved very efficiently online. The order of approximation $\nu$ affects linearly the state dimension of the MPC problem for which the optimisation problem is solved leading to a computationally tractable setting. This work paves the way for the application of model predictive control to fractional-order pharmacokinetics, as we discussed in the introduction, where satisfaction of constraints is of high importance. 

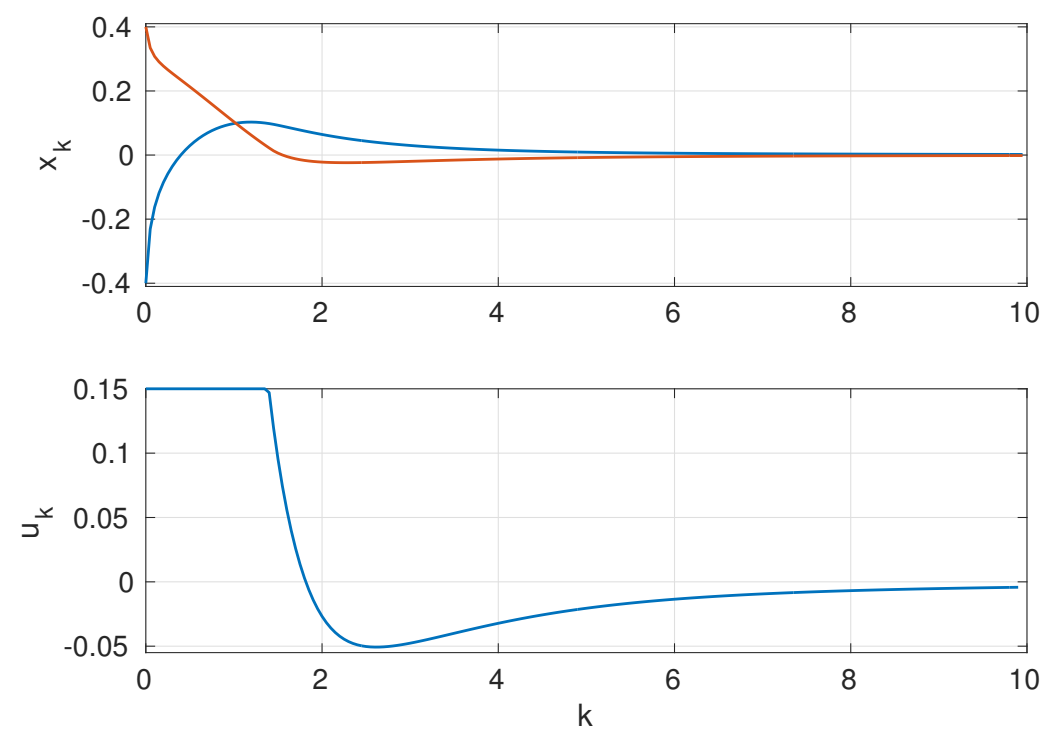

Fig. 3: Closed-loop simulations of system (34) with the proposed MPC controller.

An important question that needs to be answered is under what condition the closed loop system is asymptotically stabilising to the origin and how the MPC controller needs to be designed so as to achieve asymptotic stability to the origin. Future work will also focus on the study of sampled-data fractional-order systems, coming as an extension of [28] for linear time-invariant sustems, and applications of the proposed methodology to biomedical systems.

\section{REFERENCES}

[1] I. Podlubny, Fractional differential equations, vol. 198 of Mathematics in Science and Engineering. San Diego, California: Academic Publisher, 1999.

[2] R. Hilfer, Applications of Fractional Calculus in Physics. Singapore: World Scientific, 2000.

[3] J. Kytariolos, A. Dokoumetzidis, and P. Macheras, "Power law IVIVC: An application of fractional kinetics for drug release and absorption," European Journal of Pharmaceutical Sciences, vol. 41, no. 2, pp. 299-304, 2010.

[4] A. Dokoumetzidis and P. Macheras, "IVIVC of controlled release formulations: Physiological-dynamical reasons for their failure," Journal of Controlled Release, vol. 129, no. 2, pp. 76-78, 2008.

[5] A. Dokoumetzidis, R. Magin, and P. Macheras, "Fractional kinetics in multi-compartmental systems," Journal of Pharmacokinetics and Pharmacodynamics, vol. 37, no. 5, pp. 507-524, 2010.

[6] A. Dokoumetzidis and P. Macheras, "The changing face of the rate concept in biopharmaceutical sciences: From classical to fractal and finally to fractional," Pharmaceutical Research, vol. 28, no. 5, pp. 1229-1232, 2011.

[7] P. Sopasakis and H. Sarimveis, "Controlled drug administration by a fractional PID," in 19th IFAC World Congress, (Cape Town, South Africa), pp. 8421-8426, August 2014.

[8] M. Romero, Á. de Madrid, C. Mañoso, and R. Berlinches, "Generalized predictive control of arbitrary real order," in New Trends in Nanotechnology and Fractional Calculus Applications (D. Baleanu, Z. B. Guvenc, and J. A. T. Machado, eds.), pp. 411-418, Springer Netherlands, 2010.

[9] D. Boudjehem and B. Boudjehem, "The use of fractional order models in predictive control," in 3 rd Conference on Nonlinear Science and Complexity, symposium: Fractional Calculus Applications, (Ankara, Turkey), July 2010.

[10] Z. Deng, H. Cao, X. Li, J. Jiang, J. Yang, and Y. Qin, "Generalized predictive control for fractional order dynamic model of solid oxide fuel cell output power," Journal of Power Sources, vol. 195, no. 24, pp. 8097 - 8103, 2010.

[11] M. Romero, Á. de Madrid, C. Mañoso, and B. Vinagre, "Fractional-order generalized predictive control: Formulation and some properties," in Control Automation Robotics Vision (ICARCV), 2010 11th International Conference on, pp. 1495-1500, Dec 2010.

[12] M. Romero, Á. de Madrid, C. Mañoso, V. Milanés, and B. Vinagre, "Fractional-order generalized predictive control: Application for low-speed control of gasoline-propelled cars," Mathematical Problems in Engineering, vol. 2013, pp. 1-10, 2013. Article ID 895640.

[13] A. Rhouma, B. Bouzouita, and F. Bouani, "Model predictive control of fractional systems using numerical approximation," in Computer Applications Research (WSCAR), 2014 World Symposium on, pp. 1-6, Jan 2014.

[14] M. Romero, Á. de Madrid, C. Mañoso, and B. Vinagre, "A survey of fractional-order generalized predictive control," in Decision and Control (CDC), 2012 IEEE 51st Annual Conference on, pp. 6867-6872, Dec 2012.

[15] S. Domek, "Fuzzy predictive control of fractional-order nonlinear discrete-time systems," Acta mechanica et automatica, vol. 5, no. 2,2010 . 
[16] S. Domek, "Switched state model predictive control of fractional-order nonlinear discrete-time systems," Asian Journal of Control, vol. 15, no. 3, pp. 658-668, 2013.

[17] M. Busłowicz and T. Kaczorek, "Simple conditions for practical stability of positive fractional discrete-time linear systems," Int. J. Appl. Math. Comput. Sci., vol. 19, no. 2, pp. 263-269, 2009.

[18] S. Guerman, S. Djennoune, and M. Bettayeb, "Discrete-time fractional-order systems: Modeling and stability issues," in Advances in discrete-time systems (M. Mahmoud, ed.), Intech publications, 2012.

[19] M. Romero, I. Tejado, J. Suárez, B. Vinagre, and Á. de Madrid, "GPC strategies for the lateral control of a networked AGV," in Proc. IEEE Int. Conf. Mechatronics, 2009.

[20] S. Guermah, S. Djennoune, and M. Bettayeb, "A new approach for stability analysis of linear discrete-time fractional-order systems," in New Trends in Nanotechnology and Fractional Calculus Applications (D. Baleanu, Z. B. Guvenc, and J. A. T. Machado, eds.), pp. 151-162, Springer Netherlands, 2010.

[21] R. T. Rockafellar and R. J.-B. Wets, Variational Analysis. Grundlehren der mathematischen Wissenschaften, Dordrecht: Springer, 1998.

[22] P. Gritzmann and B. Sturmfels, "Minkowski addition of polytopes: Computational complexity and applications to Gröbner basis," SIAM J. Discrete Math., vol. 6, no. 2, pp. 246-269, 1993.

[23] A. Kurzhanski and I. Alyi, Ellipsoidal Calculus for Estimation and Control. Boston: Birkhhäuser, 1997.

[24] M. Diehl and J. Bjornberg, "Robust dynamic programming for min-max model predictive control of constrained uncertain systems," Automatic Control, IEEE Transactions on, vol. 49, pp. 2253-2257, Dec 2004.

[25] J. B. Rawlings and D. Q. Mayne, Model Predictive Control: Theory and Design. Nob Hill Publishing, 2009.

[26] D. Mayne, J. Rawlings, C. Rao, and P. Scokaert, "Constrained model predictive control: Stability and optimality," Automatica, vol. 36, pp. 789-814, 2000.

[27] J. Löfberg, "Yalmip : A toolbox for modeling and optimization in MATLAB," in Proceedings of the CACSD Conference, (Taipei, Taiwan), 2004.

[28] P. Sopasakis, P. Patrinos, and H. Sarimveis, "MPC for sampled-data linear systems: Guaranteeing continuous-time positive invariance," IEEE Trans. Auto. Contr., vol. 59, no. 4, pp. 1088-1093, 2013. 